\title{
Influence of laser wavelength and beam profile on the coagulation depth in a soft tissue phantom model
}

\author{
Martin Wehner $^{1}$ (D) Patricia Betz ${ }^{1} \cdot$ Mirko Aden $^{1}$ \\ Received: 15 September 2017 / Accepted: 18 July 2018 / Published online: 24 July 2018 \\ (C) The Author(s) 2018
}

\begin{abstract}
In laser tissue soldering (LTS), a protein solution is thermally denatured and cross-linked to obtain a strong bond between tissues or tissue and a wound dressing. However, if the extension of the heat-affected zone is too large, wound healing is impaired by thermal tissue injuries. Therefore, heat input and coagulation depth have to be limited. We investigate the influence of wavelength and beam profile on coagulation depth using a soft tissue phantom in case of weakly $(980 \mathrm{~nm})$ and strong $(1540 \mathrm{~nm})$ absorbed laser radiation. The soft tissue phantom is doped with polystyrene (PS) beads to obtain similar scattering properties as natural tissue. The propagation of the laser radiation in the phantom is simulated by Monte-Carlo method and the optical penetration (OPD) depth calculated from isophotes. The simulation results are compared with the experimental determination of the coagulation volume. The results reveal that scattering effect of tissues on laser radiation increases the losses of a Gaussian beam profile laterally leading to a half-sphere coagulation volume. The depth profile of the coagulation follows approximately the intensity distribution of the laser beams as long as scattering effects are weak. As scattering effects become significant, as for 980-nm radiation, the intensity distribution of the laser beam in the tissue deviates from the original one, leading to different profile of the coagulation depth.
\end{abstract}

Keywords Laser $\cdot$ Laser tissue soldering $\cdot$ Soft tissue phantom $\cdot$ Radiation transport in turbid media $\cdot$ Four-flux model

\section{Introduction}

Laser tissue soldering (LTS) and laser tissue welding (LTW) are two similar procedures for photothermal tissue bonding. In laser tissue welding, a thermally induced cross-linking of tissue proteins can be obtained after approximation of the edges of an incision and laser irradiation. In LTS, a protein solution is added which serves as glue or "solder" between a wound surface and a dressing or patch. The protein solution is thermally cross-linked and bonds the dressing to the tissue. Due to reduced thermal damage to tissue, LTS is often preferred over laser tissue welding [1]. Nevertheless, a thin tissue layer in the wound area is affected by the heat treatment. Laser tissue soldering is being investigated since many years. Various laser sources, solder compositions, and patches have been

Martin Wehner

martin.wehner@ilt.fraunhofer.de

1 Fraunhofer Institute for Laser Technology ILT, 52074 Aachen, Germany described in literature. For practical reasons, most procedures rely on using small form factor diode lasers in the range from 800 to $2000 \mathrm{~nm}$ that are available at reasonable prices, and beam delivery is feasible using standard quartz fibers.

In LTS, the laser radiation heats up the protein solution to a temperature above $65{ }^{\circ} \mathrm{C}$ which results in denaturation and cross-linking of the proteins, while the water content will be reduced. Typically, an irradiation time of a few seconds is sufficient to induce the structural change of albumin solder. The extension of the heat-affected zone (HAZ) can be further limited by suitable choice of wavelength and control of the optical penetration depth. A major challenge in LTS is to minimize the depth of the HAZ and to limit the heat input into tissue while ensuring a sufficiently high bonding strength.

An important measure to control heat input is to sense the surface temperature during laser irradiation. Eyal and Katzir [2] used a liquid nitrogen-cooled $\mathrm{HgCdTe}$ photonic detector which was sensitive in the $8-$ to $12-\mu \mathrm{m}$ spectral range for sensing temperature sensing during laser tissue welding. Later, the technique was extended to use a thermal camera for skin welding by Fried et al. [3]. Kirsch et al. prosed laser soldering for urologic reconstruction [4] and skin closure [5, 6] 
was demonstrated in rat model. In most of experimental work, albumin solder doped with indocyanine green (ICG) was used to enhance the absorption for 810-nm diode lasers. Other approaches used $\mathrm{CO}_{2}$ lasers and albumin solder without extrinsic absorbers for wound closure of incisions $[7,8]$ where a set point temperature of $65{ }^{\circ} \mathrm{C}$ for closing skin incision in vivo was proposed. Khosroshahi et al. reported the use of controlled feedback loop to bond rat skin successfully in vivo, the samples revealed high mechanical strength when the temperature was stabilized below $80^{\circ} \mathrm{C}$ [9]. Photobleaching of ICG lowers the absorption of laser radiation at $810 \mathrm{~nm}$ and can reduce the heat generation in a self-limiting effect. In many papers, the importance of temperature control is pointed out to reduce excessive heating, particularly, when no photobleachable chromophores are used.

Since tissues exhibit a great variety of composition and morphology, this leads to varying optical properties and susceptibility to temperature-related effects. Therefore, we decided to use a soft tissue phantom as a model for our investigations. Soft tissue phantoms as described in literature can consist of different chromophores to tailor absorption, and microparticles or lipids as scattering centers to match the desired optical properties of soft tissue. We choose a recipe according to lizuka [10] based on hen-egg albumin and agar; the advantages of the approach are low-cost, non-toxicity, good availability, and easy preparation. The phantom can be cast into any shape due to its gel-like consistency. The most important feature of the hen-egg albumin phantom is its tissue-equivalent reaction under laser radiation. The hen-egg albumin denaturizes above $60{ }^{\circ} \mathrm{C}$ similar to tissue proteins and form a cross-linked structure. The affected volume then becomes visible by blurring of the gel and strong light scattering.

Laser diodes have become cheap and convenient laser sources for medical applications, due to their compactness, reliability, and high electro-optical efficiency. Nevertheless, the undefined far-field distribution is one major drawback of diode lasers [11]. In literature, various methods for beam homogenization based on specialized optics, rearrangement of diode emitters, micro optical arrays, and optical waveguides are described. Medium power diode lasers for medical applications are usually assembled with coupling optics for directing the laser beam into an optical fiber. This leads to a nearly Gaussian beam profile with some deviations, depending on the optical system. For example, when optical stacking (spatial multiplexing) of laser diode bars is employed to couple different sources into one optical fiber, the beam from the central laser bar is transmitted with Gaussian-like beam profile, whereas radiation coming from the outer bars is formed to a donut-like beam profile [12]. The effect is observed for offaxis coupling into large core fibers.

In this work, we compare the coagulated volumes generated by two laser sources, one emitting radiation at a wavelength of $980 \mathrm{~nm}$, the other of $1540 \mathrm{~nm}$. Both wavelengths are common in laser surgery. First, a phantom model is prepared to match the absorption and scattering properties of soft tissue. Then, the depth and shape of coagulated volumes are experimentally determined and compared to a numerical simulation of radiation transport and the resulting heat generation in the phantom. To investigate the influence of the intensity distribution, we perform these procedures for a top hat and Gaussian distribution.

\section{Materials and methods}

\section{Preparation of phantom}

The soft tissue phantom is produced similar as described by Iizuka [10], except that the originally proposed naphthol green dye is not used because the dye is absorbing at $800 \mathrm{~nm}$ and therefore not effective at 980 and $1540 \mathrm{~nm}$ wavelength. First, $22.2 \mathrm{wt} \%$ of hen-egg albumin (Fluka BioChemika, 9006-591 ) is dissolved in distilled water with the help of a magnetic stirrer. The rotational speed of the stirrer is set to $200 \mathrm{rpm}$ to reduce foaming of the solution. The hen-egg albumin is given stepwise in small amounts to distilled water to prevent clotting. For the agar-agar solution distilled water is heated in a water bath up to a temperature of $70{ }^{\circ} \mathrm{C}$ firstly. At $70{ }^{\circ} \mathrm{C}, 1.4 \mathrm{wt} \%$ of agar (Amresco, J637-500G) is added to the water with a rotational speed of $200 \mathrm{rpm}$ and is heated up further until $85^{\circ} \mathrm{C}$. Afterwards, the agar solution is cooled down in a water bath to $45^{\circ} \mathrm{C}$. In the meantime, the albumin solution is heated up to $40{ }^{\circ} \mathrm{C}$ in a water bath at $200 \mathrm{rpm}$. When reaching the desired temperature, both solutions are mixed together and stirred for $5 \mathrm{~min}$ at $200 \mathrm{rpm}$ until a homogenous solution is created. To ensure a homogenous mixing of both solutions, they stirred for $5 \mathrm{~min}$ in a warm water bath to prevent the agar solution from fast solidification.

To enhance the scattering properties of the phantom, we add polystyrene beads (07310-15, Polyscience Inc., Warrington, USA) with a mean diameter of $1 \mu \mathrm{m}$ and a concentration of $8.5 \times 10^{5}$ beads $/ \mathrm{mm}^{-3}$ [13]. The phantom components (hen-egg albumin and agar) are prepared as described before, and the polystyrene suspension is added when the albumin and agar solutions are mixed together. The solution is cast then into polystyrene boxes of $10 \times 10 \times 40-\mathrm{mm}^{3}$ size; after cooling down, the samples were stored in a fridge at $4{ }^{\circ} \mathrm{C}$ for a maximum time of 4 weeks.

\section{Determination of optical properties: spectrometer and 4-flux analysis}

The optical properties of the tissue samples can be described by absorption coefficient, scattering coefficient, and scattering function. Since these properties are not directly measurable, they are obtained from transmittance and reflectance data. These data were determined on thin slices cut from blocks of 
the cast phantom. Slices of 1-2-mm thickness were manually cut from the gel phantom with cross section of $10 \times 10 \mathrm{~mm}^{2}$ using scalpels and placed in the middle of a slide. Besides the gel, two slides were positioned serving as spacers and another slide gently placed on top. By slowly pressing down the top slide, a gap of $1 \mathrm{~mm}$ totally filled with gel was obtained. The samples were placed in an UV/VIS/NIR-Spectrometer (Lambda 1050, Perkin Elmer) to measure total transmittance $T$, total reflectance $R$, and direct transmittance $T_{\mathrm{d}}$ as a function of wavelengths. The connection between the measured data and the optical properties is obtained from the solution of the radiation transfer equation in the four-flux approximation [14-17]. The first and second flux (f1, f2) correspond to the rays, which propagate parallel (f1) or antiparallel (f2) to the incoming beam without being scattered. The third and fourth flux ( $f 3, f 4)$ are built from all rays, which have been scattered in forward (f3) or backward (f4) direction relative to the incoming beam. Since scattering of the radiation is described by two fluxes, the scattering function degenerates to a number, the anisotropy factor $g(-1<g<1)$. If $g$ is positive/negative, scattering is forward/backward directed. So fluxes $\mathrm{f} 3$ and $\mathrm{f} 4$ are functions of absorption and scattering coefficient and the anisotropy factor, while fluxes $\mathrm{f} 1$ and $\mathrm{f} 2$ are functions of $\mathrm{ab}-$ sorption and scattering coefficient only $[16,17]$.

Direct transmittance $T_{\mathrm{d}}$, total transmittance $T$, and reflectance $R$ can be expressed as function of the fluxes $\mathrm{f} 1-\mathrm{f} 4$. Therefore, they are functions of the optical properties. For a sample with thickness $d$ and large lateral dimensions, the relations between the transmittance/reflectance data and the optical properties are given according to the following equations [17]:

$$
\begin{gathered}
T_{d} \approx\left(1-2 R_{s}\right) e^{-\left(c_{e} d\right)} \\
T=T_{d}+\left(1-R_{s}\right) f_{3} \\
R=R_{s}\left(1+T_{d} e^{-\left(c_{e} d\right)}\right)+\left(1-R_{s}\right) f_{4}
\end{gathered}
$$

$R_{\mathrm{S}}$ denotes the reflectance due to the sample/air boundary (since $R_{\mathrm{S}}<0.05$, quadratic terms in $R_{\mathrm{S}}$ are omitted) and $c_{\mathrm{e}}$ is the extinction coefficient $\left(c_{\mathrm{e}}=c_{\mathrm{s}}+c_{\mathrm{a}}\right)$. The dependence of $\mathrm{f}_{3}$ and $\mathrm{f}_{4}$ on the optical properties is given in [14-16]. So using the measured data of $T_{\mathrm{d}}, T$, and $R$ at a certain wavelength, one can calculate $c_{\mathrm{s}}, c_{\mathrm{a}}$, and $g$ from the equations above. The calculations have been done for phantom samples with 1.1-mm thickness for both wavelengths (see the "Results" section).

\section{Setup for laser coagulation}

Due to the variety and inhomogeneity of tissues, presetting of laser power will not yield to reliable results in thermal laser therapies. A power level appropriate for one situation may lead to insufficient treatment at another spot where the morphology and the water content of tissue is slightly different; in worst case, the same setting can lead to carbonization of tissues.
Therefore, a fixed surface temperature was chosen as boundary condition and the laser power controlled accordingly. Similar to the experiments described in [18], the experimental setup consists of a fiber-coupled multi-bar diode laser module (DILAS type M1F4S22) equipped with 980- and 1540-nm diode bars, a controller with two power supplies. The 980$\mathrm{nm}$ laser bar provides a maximal output power of $46 \mathrm{~W}$ and the 1540-nm bar of up to $11 \mathrm{~W}$. During irradiation, the surface temperature is monitored by an infrared sensor CT3 ML, Optris $\mathrm{GmbH}$, Germany, with a response time of less than $2 \mathrm{~ms}$. Since the lower limit of the temperature range is at $50{ }^{\circ} \mathrm{C}$, only higher temperatures can be detected. The temperature signal is delivered to a laser diode controller CS408, Amtron GmbH, Germany, which comprises two independent power supplies with integrated digital PID controller for both laser diode bars. The temperature signal is compared to a set point value and the laser power adjusted accordingly. The power ratio of the two laser sources can either be set to a predefined value or altered during the time course. Typically, a coagulation procedure is performed at a set point temperature of $75^{\circ} \mathrm{C}$ to prevent excess heating of tissue. Because of the short response time of the sensor and the fast control circuit, the laser power will be limited safely when the programmed temperature threshold will be exceeded.

The radiation of both bars is fed into an optical multimode (MM) fiber with a core diameter of $600 \mu \mathrm{m}$, and a collimation lens projects a spot of $5 \mathrm{~mm}$ in diameter onto the sample. According to the emission pattern of the diodes and the coupling optics, a Gaussian-like intensity distribution is obtained. For changing the beam profile, a $600 \times 600-\mu \mathrm{m}^{2}$ square core (SQ) fiber can be fitted to the laser module; the resulting spot size was $4 \times 4 \mathrm{~mm}^{2}$. The SQ fiber provided a tophat-like distribution with peak-to-peak fluctuations of less than $\pm 13 \%$ at $980 \mathrm{~nm}$ wavelength or $10 \%$ at $1540 \mathrm{~nm}$, respectively. The "Gaussian" beam profile obtained from the 600- $\mu \mathrm{m}$ MM fiber was somewhat narrowed in case of $980 \mathrm{~nm}$ and broadened for the 1540-nm source. The peak intensity of the Gaussian beam with a diameter of $6 \mathrm{~mm}$ was then $14 \%$ higher than the average of $4 \times 4-\mathrm{mm}^{2}$ top hat distribution. The power transmission was similar for both fibers with $5 \%$ uncertainty.

\section{Simulation of radiation transport and modeling of heat generation}

The propagation of laser light in the tissue is simulated with the ray tracing software OpticStudio ${ }^{\circledR}$ (http://customers. zemax.com/os/opticstudio); where for a large number of rays $\left(>10^{6}\right)$, the propagation of every single ray is monitored. The scattering process is described by the scattering probability and the angular distribution. While the scattering probability determines whether a scattering process takes place or not, the angular distribution gives the direction of the scattered ray. The input parameters are the mean free path, given by the 
Table 1 Transmittances, reflectance, and calculated optical properties derived from the tissue phantom for two wavelengths

\begin{tabular}{lllllll}
\hline Wavelength $/ \mathrm{nm}$ & $T$ & $R$ & $T_{\mathrm{d}}$ & $c_{\mathrm{s}} / \mathrm{mm}^{-1}$ & $c_{\mathrm{a}} / \mathrm{mm}^{-1}$ & $g$ \\
\hline 980 & 0.73 & 0.080 & 0.18 & 1.4 & 0.12 & 0.75 \\
1540 & 0.16 & 0.045 & 0.08 & 1.1 & 1.18 & 0.58 \\
\hline
\end{tabular}

inverse of the scattering coefficient, and the anisotropy factor $g$. For the simulation, the angular distribution is given by a Henyey-Greenstein phase function $p$ [19-21].

$$
\begin{gathered}
p(\mu)=\frac{1}{2} \frac{1-g^{2}}{\left(1+g^{2}-2 g \mu\right)^{3 / 2},} \\
\mu=\cos (\theta),-\pi<\theta<\pi,
\end{gathered}
$$

$\theta \quad$ scattering angle is a random variable.

The absorption process is modeled by an exponential decay of each ray along its propagation path. The input parameter here is the absorption coefficient. Due to the scattering process, the length of the propagation path exceeds the thickness of the sample. Therefore, scattering enhances the amount of absorbed radiation in a complicated manner. As a result of the simulations, the spatial distribution of the heat rate $Q$, generated by the light absorption, is calculated by the software.

\section{Results}

The measurement of total and direct transmittance, reflectance, and the calculation of optical properties revealed the values compiled in Table 1 . Then the derived coefficients were used for ray tracing simulation of radiant flux inside the phantom ("Simulation of radiation transport and modelling of heat generation" section). The results depicted in Fig. 1 show clear differences for radiation propagation and heat rate inside the phantom volume; the heat rate is normalized to $1-\mathrm{W}$ radiation power. In a simple approximation, the heat model represents the temperature field as well. As expected, the optical and heat penetration depth depend strongly on wavelength and beam profile.

For 980 -nm radiation, the values of the heat rate are approximately an order of magnitude smaller than for $1540 \mathrm{~nm}$, according to the different absorption coefficients for both wavelengths. The influence of the two beam profiles on the heat rate distribution depends on the ratio between the scattering and absorption coefficients. If $c_{\mathrm{s}} / c_{\mathrm{a}}$ is approximately 1 as in case of 1540-nm radiation, the Gaussian beam results in a basically hemispherical distribution, whereas the top hat profile gives a flat distribution (Fig. 1c, d). If scattering becomes more dominant $\left(c_{\mathrm{s}} / c_{\mathrm{a}} \sim 12\right)$, the difference between the two distributions becomes smaller; although, it is still visible (Fig. 1a, b).

The optical penetration depth (OPD) is defined by the distance to the surface $(z=2 \mathrm{~mm})$ where the irradiance declines by a factor 1/e (see black lines in Fig. 1). For $980 \mathrm{~nm}$, it is larger for the top hat $(\mathrm{OPD} \approx 3.1 \mathrm{~mm})$ than for the Gaussian beam $(\mathrm{OPD} \approx 2.5 \mathrm{~mm})$ because for a Gaussian beam the center radiation will be scattered to the periphery, while only a small amount is scattered from the periphery to the center. For a top hat distribution, there is also scattering from the periphery to the center region, partly compensating the scattering losses of the center radiation. In case of $1540-\mathrm{nm}$ radiation, the OPD is significantly smaller than for $980 \mathrm{~nm}$ and the same for both beam profiles (OPD $\approx 0.8 \mathrm{~mm}$ ).

Due to the different OPD of both wavelengths, the heating mechanism for both wavelengths also differs. For 980-nm radiation, a coagulation depth between 2 and $3 \mathrm{~mm}$ is in the range of the OPD. Therefore, the heat needed for the coagulation process is delivered directly by absorption of the laser light. For 1540-nm radiation, a coagulation depth in this range could only be achieved by a heat flow from the surface area
Fig. 1 Normalized heat rate $Q$ in tissue due to absorption of laser light. a $980 \mathrm{~nm}$ and Gaussian beam. b $980 \mathrm{~nm}$ and top hat beam. c $1540 \mathrm{~nm}$ and Gaussian. d $1540 \mathrm{~nm}$ and top hat distribution. Calculations are based on optical properties listed in Table 1. The sample surface is located at $z=$ $2 \mathrm{~mm}$, black lines indicate the optical penetration depth (see text), and color coding is different for each wavelength

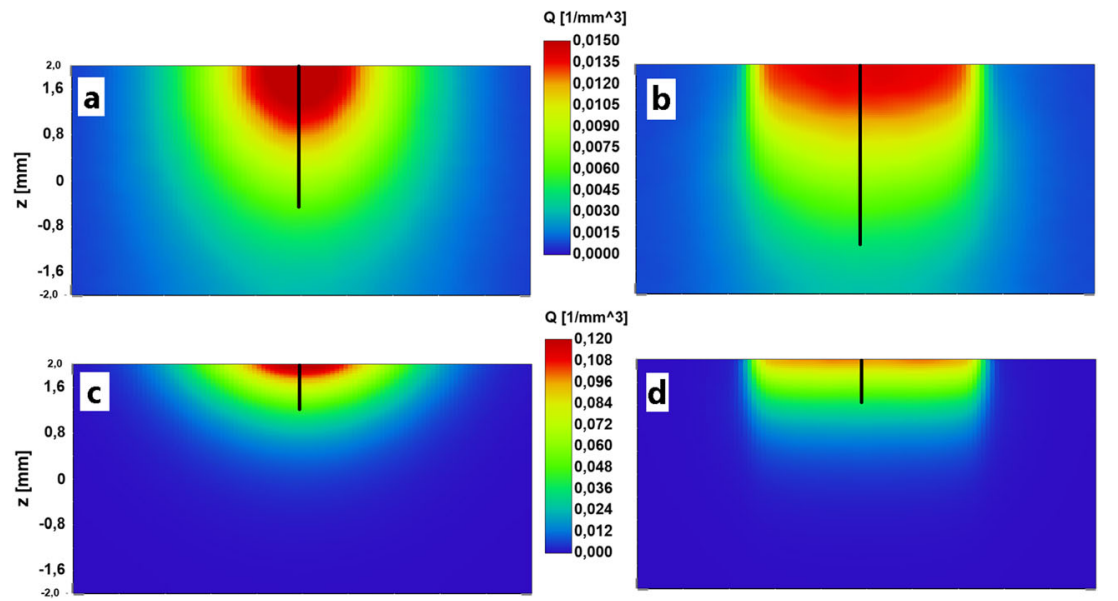


Lasers Med Sci (2019) 34:335-341

339

into the tissue. Therefore, heat conduction is more important for 1540 than for $980 \mathrm{~nm}$.

The maximum power of laser diodes used in the experiments is $46 \mathrm{~W}$ for $980 \mathrm{~nm}$ and $11 \mathrm{~W}$ for $1540 \mathrm{~nm}$. Typically, the set point temperature of $75^{\circ} \mathrm{C}$ is reached within $1 \mathrm{~s}$ for 1540 -nm radiation compared to about $2 \mathrm{~s}$ time span for 980 -nm radiation (Fig. 2). As the set point temperature is reached, the controller cuts down laser power. Despite of the lower power capability of 1540-nm source, the set point temperature rise is faster than for 980 -nm radiation. This is determined by the tenfold higher absorption coefficient in case of 1540-nm radiation.

After irradiation, the gel of the phantom gets locally crosslinked and solid clots can be carefully excavated from the gel block. After rinsing with water to remove adhering material, the clot displays the coagulated volume and its dimensions can easily be determined (see Fig. 3). The influence of the beam profile and the different penetration depth for both wavelengths are clearly visible. Irradiation experiments were performed for Gaussian and top hat beam profiles and with different laser modes. In addition to single wavelength irradiation although, combinations of both wavelengths were tested, either by simultaneous or consecutive activation. In the last case, first the 1540-nm laser sources was activated for $2 \mathrm{~s}$ and then $980 \mathrm{~nm}$ for $3 \mathrm{~s}$. Total exposure time was set to $5 \mathrm{~s}$ for all laser modes, and the maximum power settings are $46 \mathrm{~W}$ at $980 \mathrm{~nm}$ and $11 \mathrm{~W}$ at $1540 \mathrm{~nm}$, respectively.

In Fig. 4, the measured coagulation depth is compiled for each laser mode, the depth is obtained at center from 20 samplus of excavated clots. The smallest depth and most shallow shape was obtained for 1540-nm irradiation only, subsequent irradiation by $980 \mathrm{~nm}$ did not increase the depth further. Irradiation with $980 \mathrm{~nm}$ increased the coagulation to $2-3 \mathrm{~mm}$ for any beam profile, but surprisingly the same depth is obtained when a 1540-nm radiation with Gaussian profile is used.

a

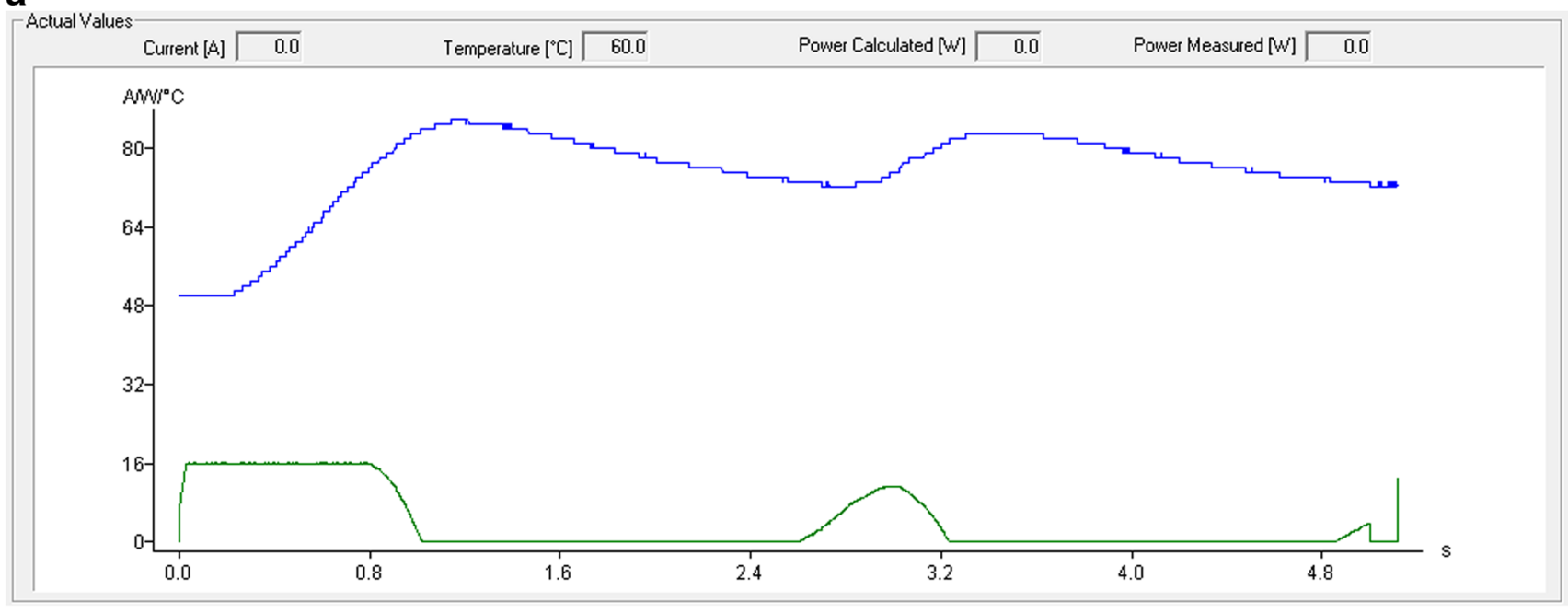

b

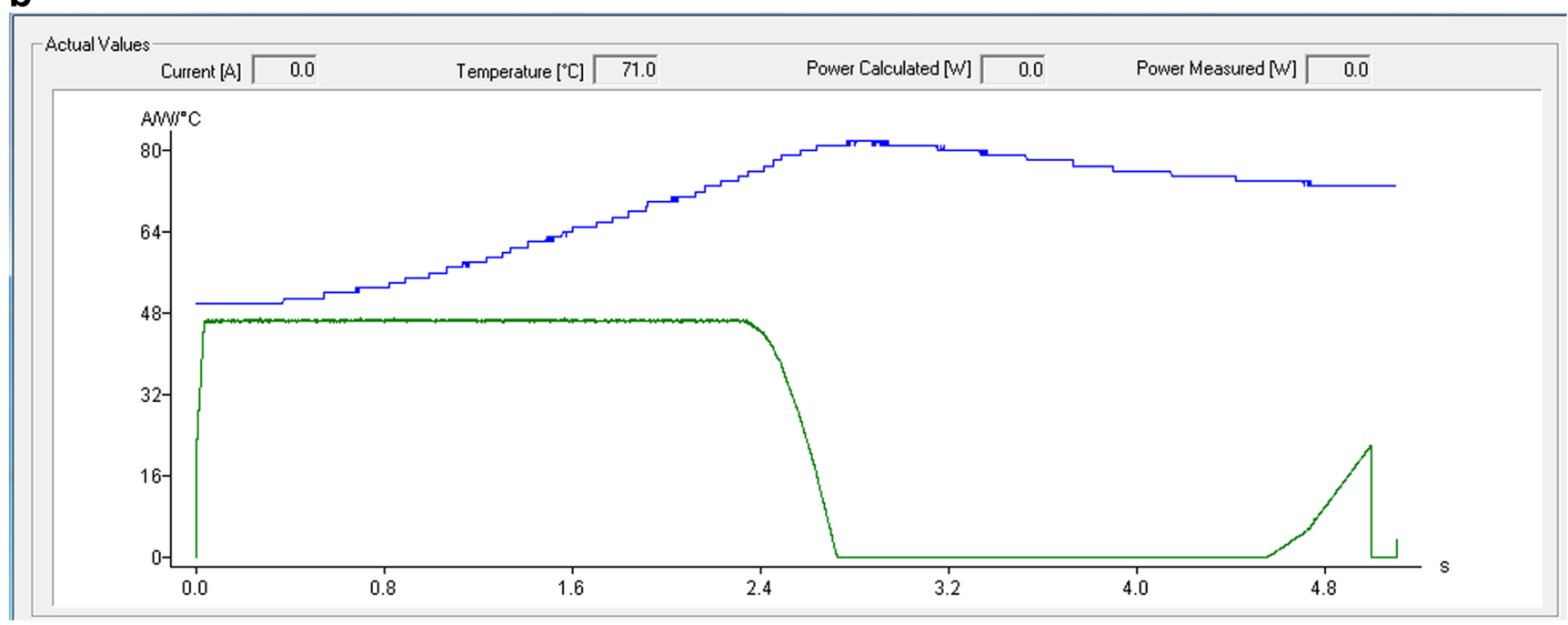

Fig. 2 Laser controller signals of the temperature sensor (upper blue line) and the laser power (lower green line) during coagulation of tissue phantom with top hat beam profile. Left, 1540 -nm radiation; right, 980 -nm radiation; set point temperature, $75^{\circ} \mathrm{C}$, and irradiation time, $5 \mathrm{~s}$ for both

Springer 
Gauss

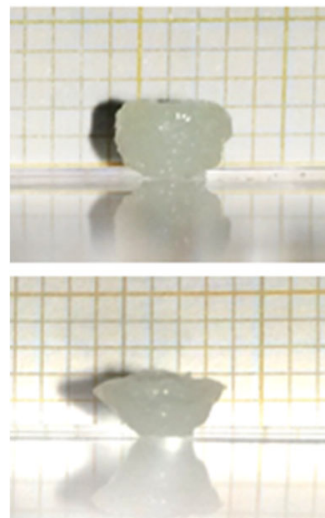

top hat

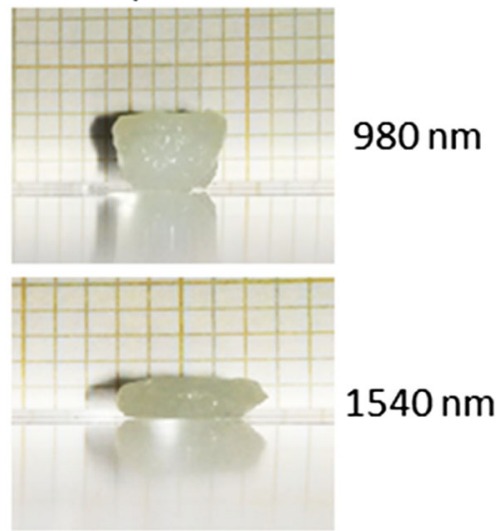

Fig. 3 Shape of coagulated clots excavated from the soft tissue phantom; the influence of wavelength and beam profile become clearly visible

The totally applied laser energy is displayed in Fig. 4 for single wavelength irradiation with either 980 - or 1540 -nm radiation. It is worthy to note that for top hat profile, the difference in coagulation depth between 980- and 1540-nm radiations is roughly a factor 2, and energy deposition at $980 \mathrm{~nm}$ is about six times higher. This indicates that for $1540 \mathrm{~nm}$, the thermal energy is concentrated in thin shallow layer introducing the structural changes of coagulation. In case of Gaussian beam profile, the coagulation depth is nearly the same for both wavelengths, but for 1540-nm radiation the shape of the coagulated volume is slimmer showing a forward peak, whereas for 980-nm radiation, the cross-sectional area is broadened due to stronger scattering (see Fig. 3). The stronger diffusion of energy is reflected by four times higher absorbed laser energy at $980 \mathrm{~nm}$ wavelength.

\section{Discussion}

For the present setup of the coagulation experiments, the results show that for the laser source with 980-nm wavelength, the coagulation depth scales with the OPD, while for the laser source with $1540 \mathrm{~nm}$, the coagulation depth exceeds the OPD. So for 980-nm radiation, the energy transport inside the phantom sample is determined by light propagation, while for $1540 \mathrm{~nm}$, it is mainly given by heat conduction. Due to the scattering process, the OPD at $980 \mathrm{~nm}$ of a Gaussian beam is smaller than for a top hat beam. For $1540 \mathrm{~nm}$, the propagation is dominated by the absorption process; therefore, the OPDs are similar for both beam profiles.

The heat rate $Q$ is a main parameter to describe the temperature field and the resulting coagulation volume. Our investigations show that for a given set of irradiation conditions (spot size, dwell time, surface temperature), the beam profile can play a major role. When the ratio of scattering and absorption coefficients $c_{\mathrm{s}} / c_{\mathrm{a}}$ is approximately 10 for 980 -nm radiation, the lateral losses are high for Gaussian profile, and the coagulation depth is slightly lower than for top hat profile. However, the shape of the volume is similar. When the absorption coefficient becomes higher $\left(c_{\mathrm{s}} / c_{\mathrm{a}} \approx 1\right.$ for $\left.1540 \mathrm{~nm}\right)$, the beam profile determines the shape of the coagulation volume. This was deduced from the model calculation and confirmed by experimental results. However, during irradiation, the gel phantom undergoes morphological changes and scattering increases. Preliminary measurements on similar gel phantoms [18] revealed a 4-fold increase of the scattering coefficient. This effects the OPD during the coagulation process but has not been taken into account here. In our model, we only calculate the generation of heat, a threshold for structural modifications, induced thermal damage, and eventually changed optical and thermal properties are not included.

Further, irradiation time can play a critical role since the temperature gradients resulting from the $3 \mathrm{D}$ distribution of the optical power density will be washed out by heat conduction for long irradiation time. The thermal relaxation time $\tau_{\mathrm{r}}$ depends on thermal diffusivity $\alpha$ and the optical penetration depth of the radiation and is calculated in the paper of Choi and Welch [22]. For our experimental conditions, we can estimate $\tau_{\mathrm{r}} \sim 1.7 \mathrm{~s}$ for $1540 \mathrm{~nm}$ and $\tau_{\mathrm{r}} \sim 15 \mathrm{~s}$ for $980-\mathrm{nm}$
Fig. 4 Coagulation depth measured at the center of clots for Gaussian (red) and top hat (black) beam profiles, the columns denote the total absorbed energy calculated from laser controller data; the error bars indicate the standard deviation calculated from 20 samples each

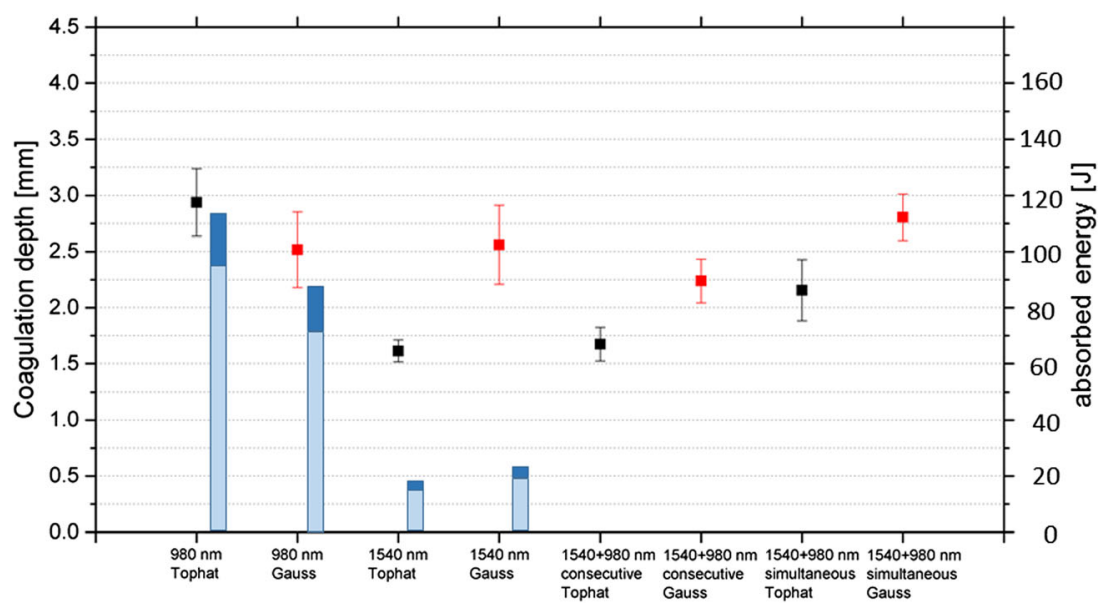


wavelength, respectively, with $\alpha=0.0015 \mathrm{~cm}^{2} / \mathrm{s}$ for the gel phantom, and OPD of $3 \mathrm{~mm}$ for $980 \mathrm{~nm}$ and $0.8 \mathrm{~mm}$ for $980-\mathrm{nm}$ radiation. The detailed analysis of heat flow by a $2 \mathrm{D}$ finite difference model in [22] leads to different correction factors depending on beam size and beam profile. The numerical results show that the effective thermal relaxation time $\tau_{\text {eff }}$ can exceed the "intrinsic" relaxation time $\tau_{\mathrm{r}}$ be substantially. Applied to our experimental conditions, we expect an effective thermal relaxation time $\tau_{\text {eff }} \sim 3.2 \mathrm{~s}$ for top hat profile and 1540-nm radiation; whereas for $980-\mathrm{nm}$ radiation and Gaussian profile, a larger value of $\tau_{\text {eff }} \sim 12 \mathrm{~s}$ is estimated. Considering our experimental dwell time of $5 \mathrm{~s}$, we conclude that in case of 980 -nm radiation, the temperature field is hardly affected by heat conduction; whereas for the stronger absorbed 1540-nm radiation, the temperature field is broadened and the depth of the coagulated zone larger than expected by only radiation transport. Therefore, heat conduction has to be taken into account in a refined model.

Even though our simple model does not include changes of optical and thermal properties and heat conduction, the main effects observed in the experiment can be explained. The experimental results and the model calculations show that a top hat beam profile is necessary to obtain a small and shallow coagulation depth when the absorption coefficient is sufficiently high as for $1540-\mathrm{nm}$ radiation, but heat conduction have to be taken into account above 3-s irradiation time. In contrast, when $980-\mathrm{nm}$ radiation is chosen for a treatment, the beam profile plays a minor role, and heat conduction can be neglected for irradiation time below $12 \mathrm{~s}$. Then the coagulation volume is dependent on radiation transport properties.

Funding information This study was supported by the EC FP7 program project BI-TRE (ERA-NET BiophotonicsPlus) 13N13173.

\section{Compliance with ethical standards}

No human participants or animals were involved in the study.

Conflicts of interest The authors declare that they have no conflicts of interest.

Open Access This article is distributed under the terms of the Creative Commons Attribution 4.0 International License (http:// creativecommons.org/licenses/by/4.0/), which permits unrestricted use, distribution, and reproduction in any medium, provided you give appropriate credit to the original author(s) and the source, provide a link to the Creative Commons license, and indicate if changes were made.

\section{References}

1. McNally-Heintzelman KM (2003) Laser tissue welding. In: Vo-Dinh $\mathrm{T}$ (ed) Biomedical photonics handbook. CRC Press, Boca Raton

2. Eyal O, Katzir A (1994) Thermal feedback control techniques for transistor-transistor logic triggered $\mathrm{CO} 2$ laser used for irradiation of bio-logical tissue utilizing infrared fiber-optic radiometry. Appl Opt 33:1751-1754

3. Fried NM, Choi B, Welch AJ et al (1999) Radiometric surface temperature measurements during dye-assisted laser skin closure: in vitro and in vivo results. Lasers Surg Med 25(4):291-303

4. Kirsch AJ, Canning DA, Zderic SA et al (1997) Laser soldering technique for sutureless urethral surgery. Tech Urol 3(2):108-113

5. Capon A, Souil E, Gauthier B et al (2001) Laser assisted skin closure (LASC) by using a 815-nm diode-laser system accelerates and improves wound healing. Lasers Surg Med 28(2):168-175. https://doi.org/10.1002/1sm.1035

6. Simhon D, Ravid A, Halpern M et al (2001) Laser soldering of rat skin, using fiberoptic temperature controlled system. Lasers Surg Med 29(3):265-273. https://doi.org/10.1002/lsm.1118

7. Brosh T, Simhon D, Halpern M et al (2004) Closure of skin incisions in rabbits by laser soldering II: tensile strength. Lasers Surg Med 35(1):12-17. https://doi.org/10.1002/1sm.20073

8. Simhon D, Gabay I, Shpolyansky G et al (2015) Temperaturecontrolled laser-soldering system and its clinical application for bonding skin incisions. J Biomed Opt 20(12):128002. https://doi. org/10.1117/1.JBO.20.12.128002

9. Khosroshahi ME, Nourbakhsh MS, Saremi S et al (2010) Application of albumin protein and indocyanine green chromophore for tissue soldering by using an IR diode laser: ex vivo and in vivo studies. Photomed Laser Surg 28(6):723-733. https://doi. org/10.1089/pho.2008.2463

10. Iizuka MN, Sherar MD, Vitkin IA (1999) Optical phantom materials for near infrared laser photocoagulation studies. Lasers Surg Med 25(2):159-169

11. Traub M, Hoffmann H-D, Plum H-D et al (2006) Homogenization of high power diode laser beams for pumping and direct applications. In: Zediker MS (ed) SPIE. 61040Q

12. Neukum J (2007) Pump up the power. Nat Photonics 1:385 EP. https://doi.org/10.1038/nphoton.2007.111

13. Polybead® Microspheres $1.00 \mu \mathrm{m}$. http://www.polysciences.com/ german/polybead-microspheres-100181m. Accessed 07 Sep 2017

14. Ishimaru A (1997) Wave propagation and scattering in random media. IEEE Press; Oxford University Press, New York, Oxford, New York

15. Mudgett PS, Richards LW (1971) Multiple scattering calculations for technology. Appl Opt 10(7):1485-1502. https://doi.org/10. 1364/AO.10.001485

16. VanGemert MJ, Welch AJ, Star WM et al (1987) Tissue optics for a slab geometry in the diffusion approximation. Lasers Med Sci 2: 295-302

17. Aden M, Roesner A, Olowinsky A (2010) Optical characterization of polycarbonate: influence of additives on optical properties. J Polym Sci B 48:451-455

18. Wehner M, Aden M, Toedter N et al (2015) Two-wavelength approach for control of coagulation depth during laser tissue soldering. In: Jansen ED (ed). SPIE, p 932109

19. Gangadhara S, Norton K (2016) Using the Henyey-Greenstein distribution to model bulk scattering. http://customers.zemax.com/os/ resources/learn/knowledgebase/using-the-henyey-greensteindistribution-to-model. Accessed 11 Jan 2018

20. Henyey LG, Greenstein JI (1941) Diffusive radiation in the galaxy. Astrophys J 93:70-83

21. Roggan A, Schädel D, Netz U et al (1999) The effect of preparation technique on the optical parameters of biological tissue. Appl Phys B 69:445-453

22. Choi B, Welch AJ (2001) Analysis of thermal relaxation during laser irradiation of tissue. Lasers Surg Med 29(4):351-359. https://doi.org/10.1002/lsm.1128 